impending synucleinopathy with over 90\% developing either Parkinson's disease(PD), Dementia with Lewy Bodies(DLB) or Multiple System Atrophy(MSA) after 15 years. This finding has stimulated efforts to actively register and track progression of such patients. Here we present experience of a biobanking program established with the aim of identifying prodromal synucleinopathies to facilitate recruitment to neuroprotective trials as they become available.

Methods Patients with iRBD were prospectively and sequentially recruited. Cross-sectional comparator groups consisting of healthy controls, idiopathic PD (within 5 years of diagnosis) and DLB were also recruited. Patients underwent a standardized assessment protocol including clinical phenotyping, neuropsychometric testing, multimodal MRI, polysomnography, quantitative electroencephalography, chronobiology (melatonin and clock gene expression profiling) and gait testing. Subjects were invited for annual and biennial review.

Results 102 patients have been recruited into the study since July 2016 including 35 patients with iRBD, 26 DLB, 19 early PD and 16 controls. 15 patients have returned for follow-up with 3 converting to a synucleinopathy(2 DLB, 1 PD). $75 \%$ of participants were able to complete all elements of assessment protocol. Preliminary evaluation of iRBD participants reveals early changes in clock gene expression (BMAL1) and subtle changes in patterns of gait compared to older controls.

Conclusions Our preliminary findings demonstrate utility and feasibility of a prodromal biobanking program within the Australian context aimed at identifying prodromal synucleinopathies. Similar models can be applied to other centers to improve access and create an extended national collaborative network.

\section{ASSESSMENT OF THE EFFICACY OF ERENUMAB DURING THE OPEN-LABEL TREATMENT (13-24 WEEKS) OF SUBJECTS WITH EPISODIC MIGRAINE WHO FAILED 2-4 PRIOR PREVENTIVE TREATMENTS: RESULTS OF THE LIBERTY STUDY}

\begin{abstract}
${ }^{1}$ Lauren Giles*, ${ }^{2}$ Uwe Reuter, ${ }^{3}$ Peter Goadsby, ${ }^{4}$ Michel Lanteri-Minet, ${ }^{5}$ Peggy Hours-Zesiger, ${ }^{5}$ Chrystel Fernandes, ${ }^{6}$ Michel Ferrari, ${ }^{5}$ Jan Klatt. ${ }^{1}$ Austin Health, Melbourne, VIC, Australia; ${ }^{2}$ Department of Neurology, Charité Universitäts Medizin, Berlin, Germany; ${ }^{3}$ University of California, San Francisco, USA; ${ }^{4}$ Pain Department, Université Côte d'Azur, Nice, France; ${ }^{5}$ Novartis Pharma AG, Basel, Switzerland; ' ${ }^{6}$ Leiden University Hospital, Leiden, Netherlands
\end{abstract}

10.1136/jnnp-2019-anzan.84

Introduction To assess efficacy of erenumab in the first three months of the open-label extension phase (OLEP; 13-24 weeks) of the LIBERTY study.

Methods In the double-blind treatment phase (DBTP), 246 patients were randomized to placebo and erenumab $140 \mathrm{mg}$ for 12 weeks, following which, patients completing that phase $(\mathrm{N}=240)$ were enrolled in OLEP, to receive monthly erenumab $140 \mathrm{mg}$. Outcomes measured monthly throughout to week 24 were achievement of at least 50\%/75\%/100\% reduction in monthly migraine days (MMD), change from DBTP baseline in MMD, monthly acute migraine-specific medication days (MSMD), Headache Impact Test (HIT-6TM) total score, everyday activities (EA) and physical impairment (PI) as measured by the Migraine Physical Function Impact Diary (MPFID).

Results Overall, 228/240(95.0\%) patients completed the 24 week visit of the OLEP. In the overall population at Week
$24,39.2 \%, 15.9 \%$ and $7.0 \%$ patients achieved $\geq 50 \% / \geq 75 \% /$ $100 \%$ reduction in MMD. The mean (standard deviation) change from DBTP baseline in MMD was -2.7(4.4) and $-1.4(3.0)$ in MSMD; and -7.6(8.0), -2.5(9.2) and -4.0 (9.0) in HIT-6TM, MPFID-PI and MPFID-EA scores respectively. Patients with continuous use of erenumab showed sustained efficacy in all outcomes assessed. Patients who switched from placebo to erenumab in the OLEP showed improvement from the first measurement at Week 16 on all outcomes assessed.

Conclusions Efficacy of erenumab was sustained throughout 24 weeks in a hard to treat patient population with multiple prior preventive treatment failures. Overall, efficacy data over 24 weeks (assessed over weeks 13-16,17-20 and 21-24) was generally in line with prior erenumab trials.

\section{ASSOCIATIONS BETWEEN COGNITIVE AND MEMORY PROBLEMS, EMPLOYMENT AND QUALITY OF LIFE: A SURVEY OF EPILEPSY PATIENTS IN AUSTRALIA}

${ }^{1}$ Jeremy M Welton*, ${ }^{2}$ Christine Walker, ${ }^{3}$ Kate Riney, ${ }^{4}$ Alvin $\mathrm{Ng},{ }^{5}$ Lisa M Todd, ${ }^{6}$ Wendyl D'Souza. ' UCB Pharma, Melbourne, VIC, Australia; ${ }^{2}$ Chronic Illness Alliance, VIC, Australia; ${ }^{3}$ Queensland Children's Hospital, QLD, Australia; ${ }^{4}$ Costello Medical Singapore Pte Ltd, Singapore; ${ }^{5}$ Epilepsy Action Australia, Sydney, NSW, Australia; ${ }^{6}$ Department of Medicine, St Vincent's Hospital, The University of Melbourne, Melbourne, VIC, Australia

\subsection{6/jnnp-2019-anzan.85}

Introduction This analysis explored relationships between memory/cognitive issues, quality of life (QoL), and employment among patients with epilepsy (PwE) in Australia.

Methods Cross-sectional surveys were completed by PwE, or caregiver proxies, recruited via the online pharmacy application MedAdvisor and Australian PwE Facebook groups from May-August 2018. Data were collected on adverse events from antiepileptic drugs (AEDs), comorbidities, epilepsy severity and management, QoL (using QOLIE-10-P total score $)^{1}$ and demographics. Descriptive statistics were stratified by employment status: employed; not looking for work (NLW); looking for work (LW); or unable to work (UW), and differences in means or distributions were analysed using chisquared, Mann-Whitney $\mathrm{U}$ or Kruskal-Wallis $\mathrm{H}$ tests. Regression models were constructed to explore associations between memory/cognition-related variables and QOLIE-10-P within each employment group.

Results 950 eligible responses reporting current AED use were included $(71 \%$ via MedAdvisor, 29\% via Facebook; $55 \%$ seizure-free for $>1$ year). Mean QOLIE-10-P score was significantly different across employment groups $(\mathrm{p}<0.001)$ : 49.61 in employed PwE $(n=493), 48.87$ in NLW $(n=227)$, 32.75 in $\operatorname{LW~}(n=52)$, and 25.97 in UW $(n=178)$. After controlling for possible confounders, presence of memory problems from AEDs was associated with a -7.50 decrease in QOLIE-10-P only among employed PwE $(p=0.002)$. The extent that PwE felt bothered by memory difficulties, however, was significantly associated with QOLIE-10-P in all employment groups; generally, as level of concern about memory difficulties increased, estimated QOLIE-10-P decreased.

Conclusions Self-reported memory problems are prevalent among PwE in Australia and may impact QoL differentially depending on employment status. UCB Pharma-sponsored. 


\section{REFERENCES}

1. Cramer JA, Perrine K, Devinsky O, Meador K. A brief questionnaire to screen for quality of life in epilepsy: The QOLIE-10. Epilepsia 1996;37:577-582.

\section{A CASE OF DELAYED POST-HYPOXIC LEUKOENCEPHALOPATHY COMPLICATING DRUG OVERDOSE}

${ }^{1}$ Min Fong*, ${ }^{1}$ Nicholas Rigby, ${ }^{2}$ Paul Pun, ${ }^{3}$ Roger Mitchell, ${ }^{1}$ Daniel Schweitzer, ${ }^{1}$ Andrew Swayne*. ${ }^{1}$ Centre for Neurosciences, Mater Hospital, Brisbane, QLD, Australia; ${ }^{2}$ Mater Health Psychiatry Services, Mater Hospital, Brisbane, QLD, Australia; ${ }^{3}$ Department of Radiology, Mater Hospital, Brisbane, QLD, Australia

10.1136/jnnp-2019-anzan.86

Introduction Delayed post-hypoxic leukoencephalopathy (DPHL) is a syndrome characterised by neurological deterioration following a period of recovery after an initial hypoxic event with striking white-matter change on magnetic resonance imaging. We present a case characterised by insidious onset and a fluctuating course of cognitive and neuropsychiatric symptoms.

Methods Single case report.

Results A 61 year old lady, with a background history of previously well managed bipolar affective disorder, was found unresponsive following an intentional overdose of temazepam and tramadol. She was hypotensive, hypoxic and required ventilatory and inotropic support. Following extubation, the patient had residual left-sided weakness and MRI confirmed a right frontal watershed infarction. A three week period of clinical improvement was followed by marked deterioration firstly with fluctuating mood and other neuropsychiatric symptoms which progressed to severe impairment of cognition and alertness. There was generalised slowing on the EEG and the CSF was unremarkable. Repeat neuroimaging undertaken on day 41 of the admission, revealed new symmetric and confluent cerebral white matter changes with high signal on the Diffusion Weighted Images (DWI) and Fluid Attenuated Inversion Recovery (FLAIR) images. The patient was managed with supportive care and sustained a clinically significant recovery (MOCA 26/30), despite ongoing cognitive impairments including working memory and deficits in social cognition including mood instability and disinhibition. Repeat neuroimaging 3 months after initial presentation revealed partial resolution of the white matter changes.

Conclusion A diagnosis of DPHL should be considered in patients with variable mood and cognition following initial improvement after a hypoxic event.

\section{DO CLINICAL NURSE SPECIALIST LED STROKE FOLLOW- UP CLINICS REDUCE POST-STROKE HOSPITAL READMISSIONS AND RECURRENT VASCULAR EVENTS?}

${ }^{1}$ Alex Kao, ${ }^{1}$ Jeremy Lanford, 'Lai-Kin Wong, ${ }^{2}$ Anna Ranta* ${ }^{1}$ Neurology, Capital and Coast District Health Board and University of Otago, Wellington, New Zealand; ${ }^{2}$ Capital and Coast District Health Board and University of Otago, Wellington, Wellington, New Zealand

\subsection{6/jnnp-2019-anzan.87}

Introduction Post-discharge stroke follow-up clinics have been associated with improved outpatient care and reduced readmission. Pre-2014 there was no consistent follow-up care offered at Wellington Hospital. Our aim was to determine whether the establishment of a clinical nurse specialist
(CNS) follow-up clinic reduced the readmission 12monthrate.

Methods This is a sequential comparison of patient admitted with stroke one year prior and one year after the clinic was established in 2013. The primary outcome was hospital 12month hospital readmission rate; main secondary outcome was recurrent vascular event. Patients were identified from the hospital discharge records and underwent detailed electronic chart review. Results were adjusted for differences in baseline characteristics.

Results We identified 874 patients; 439 pre- and 435 postnurse clinic implementation. There was no significant difference between the one-year readmission rate after the establishment of the stroke follow up clinic (adjusted $\mathrm{OR}=1.06 ; 95 \%$ $\mathrm{CI}, 0.85-1.64 ; \mathrm{p}=0.804)$ and no difference in recurrent composite vascular events at one-year (adjusted $\mathrm{OR}=1.20 ; 95 \%$ CI, 0.68-2.11; $\mathrm{p}=0.528$ ). The median (IQR) time to followup to clinic after discharge was 85 (63-98.5) days. There was a trend towards a reduction in vascular events when limiting the analysis to patients who actually attended clinic, but this trend disappeared when adjusting for baseline inter-group differences.

Conclusions There was no reduction in the one-year hospital readmission or vascular event recurrence rate for patients with stroke following the establishment of a nurse specialist led stroke follow up clinic. Earlier timed follow-up and the psychosocial value offered by these clinics requires further evaluation.

\section{RECENT TRENDS IN STROKE REPERFUSION IN NEW ZEALAND}

${ }^{1}$ Andrew Leighs, ${ }^{2}$ Anna Ranta*. 'Medicine, University of Otago, Wellington, New Zealand ${ }^{2}$ Capital and Coast District Health Board and University of Otago, Wellington, Wellington, New Zealand

\subsection{6/jnnp-2019-anzan.88}

Introduction Stroke reperfusion therapy improves patient outcomes. Here we describe recent trends in the stroke reperfusion intervention rates, treatment delays, and complications across New Zealand.

Method All 20 DHBs enter acute stroke reperfusion treatment information into a compulsory, centralized, Ministry of Health $(\mathrm{MoH})$ approved and funded Stroke Register. The data from $1 / 1 / 2018$ through $30 / 9 / 18$ was cleaned and analysed to explore trends in intervention rates, time delays, and complication rates.

Results In the nine-month study period there were a total of 544 (320 males, mean (SD) age 70.6 (14.5) years) patients thrombolysed and 193 patients underwent thrombectomy. For the January-June period, for which $\mathrm{MoH}$ stroke denominator rates were available, there was a thrombolysis rate of $10.4 \%$. The overall median time (interquartile range, IQR) for onsetto-door was 76 minutes (48-118), door-to-computed tomography (CT) scan was $23(15-36)$ minutes and CT-to-needle was 33 (20-51) minutes. The overall median (IQR) time delay from hospital arrival to thrombolysis was 59 (40-85) minutes and onset-to-needle median (IQR) time was 145 (110-196). There were a total of $26(4.8 \%)$ symptomatic intracerebral haemorrhages (sICH) for the nine-month period. This compares with a thrombolysis rate of $9.85 \%$ and door-to-needle time median of 64 minutes in 2017. 